Original article

\title{
Association between opium use and bladder cancer: A case-control study in a high risk area of Iran
}

\author{
Hamideh Rashidian ${ }^{\text {a,b }}$, Ali Akbar Haghdoost ${ }^{c}$, Maryam Hadji ${ }^{\text {d }}$, Maryam Marzban e,f, \\ Mahin Gholipour ${ }^{\mathrm{g}}$, Kazem Zendehdel ${ }^{\mathrm{a}, \mathrm{h}, \text {,* }}$ \\ ${ }^{a}$ Cancer Research Center, Cancer Institute of Iran, Tehran University of Medical Sciences, Tehran, Iran \\ ${ }^{\mathrm{b}}$ Department of Biostatistics and Epidemiology, School of Public Health, Kerman University of Medical Sciences, Kerman, Iran \\ ${ }^{\mathrm{c}}$ Regional Knowledge Hub, And WHO Collaborating Centre for HIV Surveillance, Institute for Futures Studies in Health, Kerman University of Medical Sciences, Kerman, \\ Iran \\ ${ }^{\mathrm{d}}$ Health Science Unit, Faculty of Social Sciences, Tampere University, Tampere, Finland \\ ${ }^{\mathrm{e}}$ Department of Public Health, School of Public Health, Bushehr University of Medical Science, Bushehr, Iran \\ ${ }^{\mathrm{f}}$ Clinical Research Development Center, The Persian Gulf Martyrs, BushehrUniversity of Medical Sciences, Bushehr, Iran \\ ${ }^{g}$ Golestan Research Center of Gastroenterology and Hepatology, Golestan University of Medical Sciences, Gorgan, Iran \\ ${ }^{\mathrm{h}}$ Cancer Biology Research Center, Cancer Institute of Iran, Tehran University of Medical Sciences, Tehran, Iran
}

\section{A R T I C L E I N F O}

\section{Keywords:}

Opium

Bladder cancer

Underreporting bias

Sensitivity analyses

\begin{abstract}
A B S T R A C T
Background: Bladder cancer is one of the common cancers. Currently some studies found an association between opium use and incidence of bladder cancer, however, underreporting and detection bias was high in the previous studies and also some of them did not adjust their results for confounding variables and had small sample size, various and unclear definition of opium use, and lack of data on starting age, duration, dose, and route of opium consumption. In this study we investigated the association between opium use and incidence of bladder cancer, overcoming previous studies limitations and doing sensitivity analyses for underreporting bias.

Methods: We performed a population-based case-control study, including 300 cases diagnosed with bladder cancer and 600 controls (matched for age, sex, and place of residence) between 2013-2015. We used conditional logistic regression models to estimate odds ratios (OR) and 95\% confidence intervals (CI).

Results: Overall, 200 cases (64.9\%) and 172 controls (27.9\%) reported regular use of opium, resulting in an adjusted OR (95\% CI) of 4.4 (2.9-6.5). Dose response relationship was seen and the adjusted OR for low and high dose consumption groups were 4.2 (95\% CI 2.6-6.8) and 4.5 (95\% CI 2.9-7.2) respectively. The association between opium use and bladder cancer was statistically significant even after controlling for underreporting bias. Conclusion: This study confirmed that opium use was associated with the bladder cancer incidence. We suggest primary prevention and early detection for bladder cancer, especially in the high risk groups.
\end{abstract}

\section{Introduction}

According to the United Nations Office of Drugs and Crime, about 19 million people across the world used opiates illicitly in 2016. ${ }^{1}$ Although raw opium consumption is mostly limited to some parts of Asia but local types of opiates, such as cherniashka or kompot are consumed almost exclusively in East Europe. Moreover, Heroin is the most commonly used opiate globally including Asian, American, European and African countries.

A recent systematic review showed that opium use is potential risk factor for bladder cancer. ${ }^{2}$ However, risk of underreporting and detection bias in the previous studies was high. Moreover, some of them did not adjust their results for confounding variables like smoking cigarettes, age and sex. Furthermore, previous studies about association of opium use and bladder cancer had several limitations, including small sample size, various and unclear definition of opium use, and lack of data on starting age, duration, dose, and route of opium consumption.

Bladder cancer is the 7th most common cause of cancer incidence in the world among men and it is about 4times higher in men (ASR $=9.6$ per 100,000 ) than woman (ASR $=2.4$ per 100,000) globally. Bladder cancer is the 2nd most common cancer in Iranian men (ASR $=13.4$ per 100,000). ${ }^{3}$ Kerman province, located in the southeastern part of Iran has

\footnotetext{
* Corresponding author. Cancer Research Center, Cancer Institute of Iran, Imam Khomeini Hospital, Keshavarz Bulvard, 13145-158, Tehran, Iran.

E-mail address: kzendeh@tums.ac.ir (K. Zendehdel).
} 
one of the high incidence rate of bladder cancer in Iranian men (ASR = 24.7 per 100,000$).{ }^{4}$ Due to aging, and life style changes an increasing trend of bladder cancer incidence is expected for future.

Prevalence of opium use in Iran is the highest one in the world. ${ }^{1}$ and Kerman province exhibits one of the highest prevalence for opium use in Iran ${ }^{5}$ In this study we designed a case-control study to assess the association between opium use and bladder cancer incidence. We adjusted results of the current study for under reporting bias using our validation study results ${ }^{6}$.

\section{Method}

\subsection{Cases and controls recruitment}

We conducted a case-control study in Kerman province located in the southern part of Iran. Cases included pathologically confirmed incident cases of bladder cancer (ICD-O code: C67) that were diagnosed less than one year prior to the time of the interview. We recruited our patients from newly diagnosed bladder cancer cases between 2013 and 2015.

We interviewed both cases and controls in their residential places. In order to choose suitable control for each case, considering regional municipality of cases residential place, we randomly selected six houses using simple random selection procedure. Then we referred to the house number one and asked about the age and gender of the household members and the interviewer chose one person as a potential control. If there were two or more than two potential control, the interviewer randomly selected one person. If the person was not eligible or not willing to participate in the study, the interviewer would go to the next house. We individually matched cases and controls on sex and age.

\subsection{Questionnaire}

Trained interviewers administered a structured valid and reliable questionnaire with 87 questions to each study participant. Details about development and validation of the questionnaire are described elsewhere. ${ }^{6}$ Questionnaire included questions on demographic characteristics, socioeconomic status (SES) indicators, occupational history and exposure to specific job chemical agents and history of substance use including opium; tobacco and alcohol.

Questions on opium use included regular opium use (having used at least once per week for 6 months), starting age, duration and frequency of opium use. We considered "Nokhod" as the local measurements unite of opium use which is equal to $0.2 \mathrm{~g}^{7}$. The questionnaire also included routes (ingestion and inhalation) and types of opium use (raw opium, Shireh, Sukhteh, heroin). Shireh is the condensed extract of remnants of smoked opium $^{8}$ and Sukhteh is the remnants of smoked opium. ${ }^{9}$

\subsection{Statistical analyses}

We calculated mean and standard deviations for continuous variables, and frequencies and percentages for categorical variables. We used conditional logistic regression models to calculate crude and adjusted odds ratios (OR) and 95\% confidence intervals (95\% CI). Nonusers were the referent group in all analyses. We matched cases and controls for sex, age and residential place by design. We used multivariable logistic regression analyses to adjust the results of the association between opium use and cancer for the important confounders including tobacco and alcohol use, job exposure, and SES.

We calculated cumulative use of opium by multiplying daily amount of use (in days) and duration of use (in years). We categorized participants to three groups including: non users, low and high dose users. We considered median use in the control group as cut point for categorizing participants into low and high dose groups.

In order to determine job exposure status of participants, we used International agency for research on cancer (IARC) monographs to determine carcinogenic jobs. We used principal component analyses to determine participants' socioeconomic status considering years of education and ownership of some assets including washing machine, personal car, personal computer, furniture, split, freezer and cloths wash and In order to overcome reverse causality bias, we dropped from analysis any opium use during 1,3 , and 5 years prior to diagnosis respectively, as some patients may use opium to relive their pains.

We conducted sensitivity analyses to adjust odds ratio of the association between opium use and incidence of bladder cancer for possibly underreporting bias. According to our validation study, sensitivity of self-reported opium use was 77\% (CI: $65.8 \%-89.2 \%$ ) in the hospitalized patients and 69\% (CI: 52.8\%-84.9\%) in the healthy individuals. ${ }^{6}$ Being more conservative, In order to overcome such underreporting bias, we calculated odds ratio for different sensitivities of self-reporting in cases and controls using excel 2017 for crude odds ratio. For cases we considered a range of $0.7-1$ for self-reporting sensitivity and for controls we considered a range of $0.5-1$ (Fig. 1).

We conducted all Statistical analyses with Stata Statistical Software, version 13 (STATA Corp, College Station, TX).

\subsection{Ethical considerations}

The ethics committee of Kerman University of Medical Sciences approved the study (Ethical code: 9421). All participants signed written informed consents and the data was handled confidentially. Participants received a small gift after interview.

\section{Result}

Non-Response rate in cases and controls were approximately the same and equal to about $10 \%$. "There were no statistical differences between age, gender, education and marital status of participants and non-respondents."

Demographic characteristics for cases and controls are presented in Table 1 . The mean (standard deviation) age for cases and controls were $62( \pm 11.2)$ and $64.2( \pm 10.7)$ years respectively. The average years of participants schooling were about 6 years, which was less than high school education, and about $87 \%$ of participants were males. About $60 \%$ of participants were from capital city of the Kerman province. Socioeconomic status of cases and controls was not statistically different. About $64 \%$ of cases and $41 \%$ of controls were regular cigarettes smoker. Regular alcohol use prevalence among cases was $12.7 \%$ and $8.4 \%$ among controls.

Overall, 200 (64.9) cases and 172 (27.9\%) controls reported regular opium use. Raw opium was the common type of opium used in both cases (54.5) and controls (26) and it was statistically higher in cases than controls. Also smoking was the common route of opium use and it was statistically higher in cases (54) than controls (24.2) (Table 2).

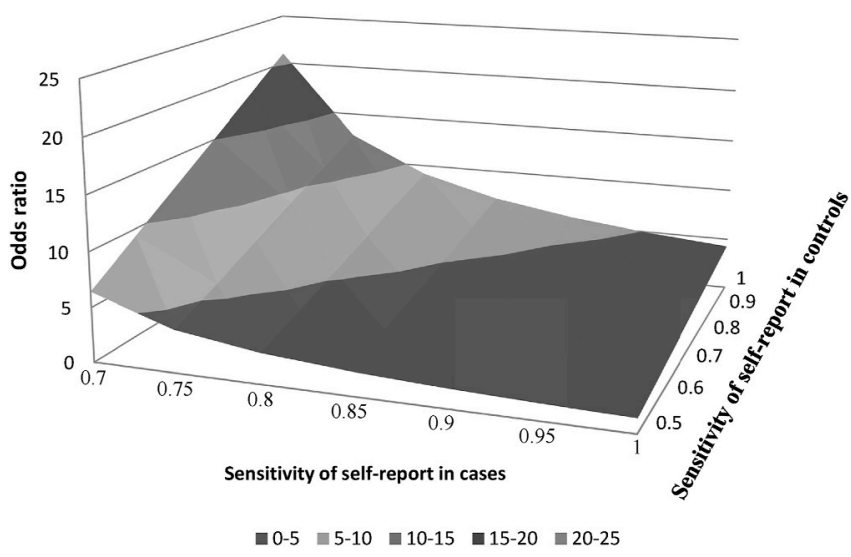

Fig. 1. Sensitivity analyses of underreporting bias for the association between opium use and bladder cancer incidence. 
Table 1

Demographic characteristic and habits of bladder cancer cases and controls.

\begin{tabular}{|c|c|c|c|}
\hline & Cases N (\%) & Controls N (\%) & $\mathrm{P}$ \\
\hline \multicolumn{4}{|l|}{ Total } \\
\hline Gender & & & 1 \\
\hline Male & $270(87.7)$ & $540(87.7)$ & \\
\hline Female & $38(12.3)$ & $76(12.3)$ & \\
\hline \multicolumn{4}{|l|}{ Place of residence } \\
\hline Capital city & $185(60.1)$ & $370(60.1)$ & 1 \\
\hline Other cities & $123(39.3)$ & 246 (39.9) & \\
\hline \multicolumn{4}{|c|}{ Socio economic status } \\
\hline low & $307(49.8)$ & $309(50.2)$ & 0.5 \\
\hline high & $143(46.4)$ & $165(53.6)$ & \\
\hline \multicolumn{4}{|l|}{ Cigarette smoking } \\
\hline Nonuser & $109(35.4)$ & 363 (58.9) & $<0.001$ \\
\hline Regular user & 199 (64.4) & $253(41.1)$ & \\
\hline \multicolumn{4}{|l|}{ Alcohol drinking } \\
\hline Nonuser & $269(87.3)$ & $564(91.6)$ & 0.04 \\
\hline \multirow[t]{2}{*}{ Regular user } & $39(12.7)$ & $52(8.4)$ & \\
\hline & Mean (SD) & Mean (SD) & \\
\hline Age (year) & $62(11.2)$ & $64.2(10.7)$ & 0.004 \\
\hline Education (year) & $6.9(5.5)$ & $6.6(5.7)$ & 0.5 \\
\hline
\end{tabular}

We found that the odds of bladder cancer was 4.4 higher among regular opium users compared to non- opium users (OR $=4.4,95 \% \mathrm{CI}$ 2.9-6.5). Dose-response relationship was seen and the association was higher among high dose opium consumption groups (OR: 4.5, 95\% CI: 2.9-7.2) compared to the low dose opium consumption group (OR: 4.2, 95\% CI 2.6-6.8) (Table 2).

Both common types of opium including Raw opium (OR: 4.2, 95\% CI 2.8-6.4) and Shireh (OR: 9.8, 95\% CI 4.1-23.3) was strongly associated with bladder cancer. The association between route of opium use and bladder cancer was statistically significant for oral ingestion (OR: 4.2, 95\% CI 1.7-10.4), smoking (OR 4, 95\% CI 2.7-6) and both routes (OR 5.9, 95\% CI 2.3-15.5) (Table 2).

As some cancer patients used opium to relive their pain, we assumed the cancer cases that started opium use within 1,3 , and 5 years prior to diagnosis as nonusers and we observed significant association for all above conditions and also dose-response relationship was seen. Ignoring the opium use 1,3 , and 5 years before diagnosis and considering the cases as nonuser, adjusted ORs were changed to $3.6(0.95 \% \mathrm{CI} 2.5-5.3)$, 3.4 (0.95\% CI 2.3-5) and 2.9 (0.95\% CI 2-4.2) respectively (Table 2).

According to the result of sensitivity analyses, In all of the scenarios mentioned in the method section the association between opium use and bladder cancer incidence was significant even in the worst scenario, when we considered self-report sensitivity of 1 for cases and 0.5 for controls (OR 1.3, 95\% CI 1-1.7) (Fig. 1).

\section{Discussion}

In this study we investigated the association between opium use and bladder cancer. We found that opium use had statistically significant association with bladder cancer even after controlling for reverse causality, potential underreporting bias and important potential confounders.

The odds ratio for opium use after underreporting bias adjustment was about 5.7 (95\%CI 4.3-7.6). Several studies have so far reported the association between opium use and bladder cancer, the reported ORs ranged between 2.6 and 8.0 and pooled OR was 5.3 (95\% CI 3.6-7.7). However about $40 \%$ of these studies were at high risk of underreporting bias and none of them adjusted their results for underreporting bias. ${ }^{2}$ About $50 \%$ of previous studies had high risk of detection bias (blinding outcome/exposure assessment, valid and reliable assessment of outcome/exposure/confounder, and similar time intervals between exposure and outcome in cases and controls), according to the Cochrane risk of bias tool. ${ }^{2}$

We adjusted our results for possible underreporting bias ${ }^{6}$ using
Table 2

The association of opium use and the bladder cancer.

\begin{tabular}{|c|c|c|c|c|}
\hline & $\begin{array}{l}\text { Cases N } \\
(\%)\end{array}$ & $\begin{array}{l}\text { Controls N } \\
(\%)\end{array}$ & $\begin{array}{l}\text { Unadjusted OR } \\
(95 \% \mathrm{CI})\end{array}$ & $\begin{array}{l}\text { Adjusted } \mathrm{OR}^{\mathrm{a}} \\
(95 \% \mathrm{CI})\end{array}$ \\
\hline \multicolumn{5}{|l|}{ Opium use } \\
\hline Non user & $\begin{array}{l}108 \\
(35.1)\end{array}$ & $444(72.1)$ & Reference & Reference \\
\hline $\begin{array}{l}\text { Regular } \\
\text { user }\end{array}$ & $\begin{array}{l}200 \\
(64.9)\end{array}$ & $172(27.9)$ & $5.3(3.7-7)$ & $4.4(2.9-6.5)$ \\
\hline $\begin{array}{l}\text { Low } \\
\text { dose }^{b}\end{array}$ & $\begin{array}{l}88 \\
(28.6)\end{array}$ & $87(14.1)$ & $5.1(3.2-8)$ & $4.2(2.6-6.8)$ \\
\hline $\begin{array}{l}\text { High } \\
\text { dose }\end{array}$ & $\begin{array}{l}112 \\
(36.4)\end{array}$ & 85 (13.8) & $5.5(3.6 .3-8.5)$ & $4.5(2.9-7.2)$ \\
\hline \multicolumn{5}{|c|}{ Type of opium used } \\
\hline Nonuser & $\begin{array}{l}108 \\
(35.1)\end{array}$ & $444(72.1)$ & Reference & Reference \\
\hline $\begin{array}{l}\text { Raw } \\
\text { opium }\end{array}$ & $\begin{array}{l}168 \\
(54.5)\end{array}$ & $160(26)$ & $4.8(3.3-7)$ & $4.2(2.8-6.4)$ \\
\hline Shireh & $\begin{array}{l}32 \\
(10.4)\end{array}$ & $12(1.9)$ & $11.5(5.1-26)$ & $9.8(4.1-23.3)$ \\
\hline \multicolumn{5}{|c|}{ Route of opium used } \\
\hline Non user & $\begin{array}{l}108 \\
(35.6)\end{array}$ & $444(72.1)$ & Reference & Reference \\
\hline Smoking & $\begin{array}{l}165 \\
(54.5)\end{array}$ & $145(23.5)$ & $4.7(3.3-6.7)$ & $4(2.7-6)$ \\
\hline Ingestion & $14(4.6)$ & $15(2.4)$ & $5.1(2.2-12.1)$ & $4.2(1.7-10.4)$ \\
\hline Both & $16(5.3)$ & 12 (1.9) & $5.9(2.4-14.3)$ & $5.9(2.3-15.5)$ \\
\hline \multicolumn{5}{|c|}{ Opium use $(1 \mathrm{yd})^{\mathrm{c}}$} \\
\hline Nonuser & $116(38)$ & $444(72.1)$ & Reference & Reference \\
\hline $\begin{array}{l}\text { Regular } \\
\text { user }\end{array}$ & $192(62)$ & $172(27.9)$ & $4.5(3.1-6.4)$ & $3.6(2.5-5.3)$ \\
\hline $\begin{array}{l}\text { Low } \\
\text { dose }\end{array}$ & $80(26)$ & $87(14.1)$ & $4(2.5-6.1)$ & $3.2(2-5.2)$ \\
\hline $\begin{array}{l}\text { High } \\
\text { dose }\end{array}$ & $112(36)$ & $85(13.8)$ & $5(3.3-7.6)$ & $4(2.5-6.3)$ \\
\hline \multicolumn{5}{|c|}{ Opium use $(3 y d)^{c}$} \\
\hline Nonuser & $\begin{array}{l}121 \\
(39.3)\end{array}$ & $444(72)$ & Reference & Reference \\
\hline $\begin{array}{l}\text { Regular } \\
\text { user }\end{array}$ & $\begin{array}{l}187 \\
(60.7)\end{array}$ & $172(28)$ & $4.2(3-6)$ & $3.4(2.3-5)$ \\
\hline $\begin{array}{l}\text { Low } \\
\text { dose }\end{array}$ & $\begin{array}{l}76 \\
(24.7)\end{array}$ & 87 (14) & $3.5(2.3-5.5)$ & $2.9(1.8-4.6)$ \\
\hline $\begin{array}{l}\text { High } \\
\text { dose }\end{array}$ & $111(36)$ & $85(14)$ & $4.7(3.1-7.2)$ & $3.8(2.4-6)$ \\
\hline \multicolumn{5}{|c|}{ Opium use $(5 y d)^{c}$} \\
\hline Nonuser & $\begin{array}{l}131 \\
(42.5)\end{array}$ & $444(72.1)$ & Reference & Reference \\
\hline $\begin{array}{l}\text { Regular } \\
\text { user }\end{array}$ & $\begin{array}{l}177 \\
(57.5)\end{array}$ & $172(27.9)$ & $3.6(2.6-5.1)$ & $2.9(2-4.2)$ \\
\hline $\begin{array}{l}\text { Low } \\
\text { dose }\end{array}$ & $\begin{array}{l}66 \\
(21.4)\end{array}$ & $87(14.1)$ & $2.8(1.8-4.4)$ & $2.3(1.4-3.7)$ \\
\hline $\begin{array}{l}\text { High } \\
\text { dose }\end{array}$ & $\begin{array}{l}111 \\
(36.1)\end{array}$ & 85 (13.8) & $4.3(2.9-6.5)$ & $3.4(2.2-5.3)$ \\
\hline
\end{tabular}

${ }^{\text {a }}$ Adjusted for age, sex, residential place, cigarette smoking, alcohol use and job exposure.

${ }^{\mathrm{b}}$ We considered median use in controls as cut point.

c 1yd, 3yd, 5yd: After assuming opium users who started opium use within 1 year, 3 year and 5 year prior to diagnosis as nonusers respectively.

validation study results. Moreover experienced interviewers were specially trained for this study in a two-day workshop to standardize the interviewing process and mitigate inter-rater variability. Also a detailed questionnaire guideline was used to decrease the risk of detection bias.

We adjusted our results for the most important potential confounders and we managed to show a dose-response association between opium use and bladder cancer incidence. We adjusted our results for reverse causality bias, ${ }^{10}$ as people may use opium to mitigate their pain in early stages of cancer. The results did not change materially after these adjustments and the associations remained statistically significant.

There are some possible mechanisms that justify the causal relationship between opium use and the occurrence of bladder cancer. 
Nitrogen-containing heterocyclic compounds are the main mutagenic compounds of opium pyrolysates. ${ }^{11}$ Also alkaloid components of opium can induce urinary retentions which prolong the exposure to other carcinogenic agents. ${ }^{12}$ Furthermore opium smoke produces polycyclic aromatic hydrocarbons that are known carcinogen and cause different cancers including bladder cancer. ${ }^{11}$

In this study we found that opium use is one of the most important preventable risk factor for bladder cancer incidence. Consequently, in high prevalent opium use areas, the role of this factor in the development of bladder cancer is not ignorable and in cancer control health policy makings, we should plan for Primary prevention strategies in high risk regions like Kerman province. Moreover we should plan for early detection program in opium users.

This study had several strengths. First, we recruited incident cases of bladder cancer and tried to control for various confounding factors. Moreover, we adjusted the results for potential underreporting bias and reverse causality bias. It had some limitations too as Opium use is an illegal behavior in Iran and people are less prone to report their consumption. However we used trained and experienced interviewers to make participants more confident to report their opium use, also we did information bias sensitivity analyses to adjust the results of the study for possibly underreporting bias.

There is an old belief among lay people and few older physicians in western and central Asia that long-term use of opium at the low dose level can prevent or cure chronic diseases like diabetes mellitus, cancer and cardiovascular diseases. ${ }^{13}$ We should inform the general population chronic complications of opium use through mass media. Moreover in planning cancer control programs we should pay special attention to opium use as an important risk factor for bladder cancer incidence. Also we should evaluate cost effectiveness of bladder cancer screening program in opium users.

In conclusion, this study confirmed that opium use is associated with the bladder cancer incidence. We could show that the association is not due to underreporting bias which is an important bias threaten the study results of sensitive issues. Moreover we showed the dose-response relationship between opium use and bladder cancer incidence. According to the current study results, opium use is one of the most important preventable risk factor and removal of this risk factor will have a significant role in bladder cancer control.

\section{Declaration of competing interest}

The authors declare that there is no conflict of interests.

\section{Acknowledgement}

This work was supported by Kerman Neuroscience Research Center, Institute of Neuropharmacology (grant number: 9421) and National Institute for Medical Research Development (grant number: 940045). Design and Most part of the questionnaire used in the current study were developed through pilot phase of the Iran Opium Cancer study (IROPICAN).

\section{References}

1 United Nations Office on Drugs and Crime. World Drug Report. 2018.

2 Afshari M, Janbabaei G, Bahrami MA, Moosazadeh M. Opium and bladder cancer: a systematic review and meta-analysis of the odds ratios for opium use and the risk of bladder cancer. PloS One. 2017;12(6), e0178527.

3 Bray F, Ferlay J, Soerjomataram I, Siegel RL, Torre LA, Jemal A. Global cancer statistics 2018: GLOBOCAN estimates of incidence and mortality worldwide for 36 cancers in 185 countries. CA A Cancer J Clin. 2018;68(6):394-424.

4 Shahesmaeili A, Afshar RM, Sadeghi A, Bazrafshan A. Cancer incidence in kerman province, southeast of Iran: report of an ongoing population-based cancer registry, 2014. Asian Pac J Cancer Prev APJCP: Asian Pac J Cancer Prev APJCP. 2018;19(6): 1533.

5 Nikfarjam A, Shokoohi M, Shahesmaeili A, et al. National population size estimation of illicit drug users through the network scale-up method in 2013 in Iran. Int J Drug Pol. 2016;31:147-152.

6 Rashidian H, Hadji M, Marzban M, et al. Sensitivity of self-reported opioid use in case-control studies: healthy individuals versus hospitalized patients. PloS One. 2017; 12(8), e0183017.

7 Shakeri R, Malekzadeh R, Etemadi A, et al. Opium: an emerging risk factor for gastric adenocarcinoma. Int J Canc. 2013;133(2), 455-463.

8 Amin-Esmaeili M, Rahimi-Movaghar A, Sharifi V, et al. Epidemiology of illicit drug use disorders in Iran: prevalence, correlates, comorbidity and service utilization results from the Iranian mental health survey. Addiction. 2016;111(10), 1836-1847.

9 Mohammadpoorasl A, Fakhari A, Rostami F, Vahidi R. Predicting the initiation of substance abuse in Iranian adolescents. Addict Behav. 2007;32(12):3153-3159.

10 Schulz KF, Grimes DA. Case-control studies: research in reverse. Lancet. 2002;359 (9304):431-434.

11 Malaveille C, Friesen M, Camus A-M, et al. Mutagens produced by the pyrolysis of opium and its alkaloids as possible risk factors in cancer of the bladder and oesophagus. Carcinogenesis. 1982;3(5):577-585.

12 Verhamme KM, Sturkenboom MC, Stricker BHC, Bosch R. Drug-induced urinary retention. Drug Saf. 2008;31(5):373-388.

13 Kamangar F, Shakeri R, Malekzadeh R, Islami F. Opium use: an emerging risk factor for cancer? Lancet Oncol. 2014;15(2):e69-e77. 